\title{
Factores asociados prospectivamente a sintomatología depresiva en adolescentes tempranos de sexo femenino*
}

\author{
Factors Prospectively Associated with of Depressive \\ Symptomatology in Early Female Adolescents
}

Recibido: abril 5 de 2010 | Revisado: octubre 14 de 2011 | Aceptado: marzo 14 de 2012

\author{
FÉlIX COVA ** \\ PAULINA Rincón *** \\ CAROLINA INOSTROZA $* * * * *$ \\ ROBERTO MELIPILLÁN ****** \\ Universidad de Concepción, Chile
}

SICI: 1657-9267(201209)11:3<853:FAPSDA>2.0.TX;2-B

Para citar este artículo. Cova, F., Rincón, P., Inostroza, C. \& Melipillán, R. (2012). Factores asociados prospectivamente a sintomatología depresiva en adolescentes tempranos de sexo femenino. Universitas Psychologica, 11 (3), 853-862.

* Conicyt Proyecto FONDECYT N ${ }^{\circ} 11060059$

** Dr. en Psicología. Profesor Asociado, Departamento de Psicología. Email: fecova@udec.cl

**** Dr. en Psicología. Profesor Asistente, Departamento de Psicología. Email: princon@udec.cl

******* Magíster en Investigación Social, Universidad del Desarrollo, Departamento de Psicología. Email: caroinostroza@udec.cl

${ }^{* * * * * * *}$ Magíster en Psicología, Universidad del Desarrollo. E-mail: rmelipillan@udd.cl

\section{RES UMEN}

Este estudio analiza prospectivamente la relación entre soporte familiar, estilo inferencial depresogénico y rumiación negativa con sintomatología depresiva en adolescentes. Se focaliza en adolescentes de sexo femenino, donde la depresión es más prevalente. Participaron 142 estudiantes de primer año de secundaria, con un promedio de edad de 14.2 años. Las evaluaciones de seguimiento fueron a 6, 13 y 24 meses de la primera evaluación. Controlada la depresión inicial, se observó relación entre soporte familiar y estilo inferencial depresogénico con sintomatología depresiva. Los resultados respaldan las teorías cognitivas de la depresión e indican la importancia de considerar estos factores en los programas preventivos de depresión.

Palabras claves autores

Adolescencia, depresión, estilo inferencial, rumiación, soporte familiar.

Palabras claves descriptores

Psicología de la salud, promoción y prevención, investigación longitudinal.

\footnotetext{
A B S T R A C T

Family support, depressogenic inferential style and rumination were longitudinally analysed in a sample of female adolescents wherein depression is more prevalent compared to adolescente male population. The sample comprised a group of 142 first grade secondary school students, with an average age of 14.2 years. The assessment was undertaken 6, 13 and 24 months after the first evaluation. Controled the initial depression, results indicated a relationship between depressive symptomatology with family support and depressogenic inferential style. These results support the cognitive theories of depression and indicate the importance of considering these factors in depression preventive programs.

Key words authors

Adolescence, Depression, Inferential Style, Rumination, Brooding, Family Support. Key words descriptors

Health Psychology, Promotion and Prevention, Longitudinal Research.
} 


\section{Introducción}

La depresión es uno de los problemas de salud mental que genera más preocupación en la actualidad a nivel mundial, debido a diversos factores: sus altas tasas de incidencia y prevalencia, el grado en que afecta la calidad de vida de las personas, y sus consecuencias sociales, sanitarias y económicas (Üstun, Ayuso-Mateos, Chatterji \& Mathers, 2004). Se estima que en el año 1990 la depresión habría sido el cuarto determinante más importante de discapacidad en el mundo y se proyecta que en el año 2020 será la segunda causa global de discapacidad, solo después de las enfermedades cardiovasculares (Murray \& López, 1998).

Por depresión se puede entender tanto la presencia de sintomatología o ánimo depresivo como la presencia de trastornos depresivos propiamente tales, existiendo evidencia que el ánimo y trastornos depresivos pueden ser vistos como parte de un continuo (Hyde, Mezulis \& Abramson, 2008). En este artículo se utilizará el término depresión para referirse tanto a ánimo depresivo como a trastornos depresivos propiamente tales (en particular, a los "monopolares").

La prevalencia de la depresión se incrementa en forma acusada en la etapa de finalización de la niñez e inicio de la adolescencia (Angold \& Costello, 2001). La multicausalidad de la depresión y la estrecha interrelación entre los cambios biológicos, psicológicos, de roles y contexto social que se dan en esta etapa de la vida ha dificultado la identificación de los factores específicos que están involucrados en dicho incremento (Brent \& Weersing, 2008).

La prevalencia de la depresión presenta una desigual distribución por género. A partir de los trece años aproximadamente la incidencia de la depresión es notoriamente más elevada en el sexo femenino (Hankin, Weeter \& Cheely, 2008; Lewinsohn, Rohde \& Seeley, 1998). Diferentes teorías intentan explicar esta mayor vulnerabilidad femenina a la depresión, existiendo autores que enfatizan sobre todo aspectos biológicos, y otros, aspectos psicosociales (Hankin et al., 1998; Hilt \& NolenHoeksema, 2009; Wade, Cairney \& Pevalin, 2002).
Diversas líneas de investigación se han centrado en procesos cognitivos y estrategias de afrontamiento como aspectos de relevancia para comprender la etiología de la depresión, considerando variables como: esquemas desadaptativos y actitudes disfuncionales, baja autoestima, estilos inferenciales y atribucionales depresogénicos, afrontamiento pasivo y rumiativo (Abela \& Hankin, 2009; Vázquez, Hervás, Hernangómez \& Romero, 2010). Algunas de estas variables podrían contribuir a explicar la mayor frecuencia de depresión en mujeres que en hombres, dado que se ha observado que se presentan con mayor frecuencia o intensidad en el género femenino (Hankin, 2009; Hilt \& Nolen-Hoeksema, 2009).

La presente investigación se concentra en dos de estas variables: estilo inferencial depresogénico (también denominado estilo cognitivo negativo) y rumiación negativa. Además de estos factores individuales de vulnerabilidad, la presente investigación considera el soporte familiar, dada su relevancia en el bienestar emocional en la adolescencia.

La existencia de estilos atribucionales depresogénicos (internos, estables y globales para los eventos negativos) que pueden favorecer el desarrollo de la depresión ha sido explorada en diversas investigaciones desde que Abramson, Seligman y Teasdale (1978) propusieron su reformulación de la teoría de la depresión por desamparo aprendido. Algunos estudios han mostrado que un estilo atribucional negativo acentúa la vulnerabilidad a desarrollar síntomas y episodios depresivos futuros (Southall \& Roberts, 2002), pero otros han fracasado en replicar dichos hallazgos (Abela \& Hankin, 2008). Esta teoría fue ampliada nuevamente por Abramson, Metalsky y Alloy (1989), planteándose como factor de riesgo de depresión la existencia de cogniciones negativas que conformarían un estilo inferencial depresogénico, el que a su vez causaría desesperanza y un subtipo particular de depresión, la depresión por desesperanza. Este estilo considera tres tipos de inferencias negativas que los individuos pueden hacer frente a la ocurrencia de eventos negativos: a) estilo atribucional generalizado negativo de causalidad (atribuciones estables y globales); b) inferencias negativas acerca de las consecuencias futuras que implica el evento, y c) inferencias nega- 
tivas de sus implicaciones para la propia persona. Investigaciones longitudinales han mostrado evidencias favorables a este planteamiento en adultos y también en adolescentes (Abela \& Seligman, 2000; Hankin, 2009).

Limitaciones psicométricas de los instrumentos empleados han dificultado la investigación respecto de la influencia del estilo atribucional, y del estilo inferencial en general en el desarrollo de depresión en niños y adolescentes. Hankin y Abramson (2002) desarrollaron recientemente un instrumento que ha mostrado propiedades psicométricas superiores a las de los instrumentos habitualmente utilizados en adolescentes. La presente investigación utiliza una versión abreviada de dicho instrumento, que conserva su elevada consistencia interna.

La existencia de un estilo de afrontamiento rumiativo, entendido como una tendencia a focalizar la atención en el estado de malestar, ha sido propuesto como un factor que contribuye al desarrollo de sintomatología depresiva (Nolen-Hoeksema, 2000). En los últimos años se ha realizado un esfuerzo por diferenciar subtipos de afrontamiento rumiativo, habiendo adquirido particular relevancia la distinción entre una tendencia a analizar las vivencias (reflexión) y otra a focalizar reiteradamente la atención en el malestar (brooding; se le traducirá como rumiación negativa) (Cova, Rincón \& Melipillán, 2007). Existen algunas evidencias respecto a que la rumiación negativa sería un factor que efectivamente incidiría en el desarrollo de sintomatología depresiva, en cambio la reflexión sería más bien un correlato de ésta (Cova, Rincón \& Melipillán, 2009; Treynor, González \& NolenHoeksema, 2003). De allí que esta investigación se focaliza en la rumiación negativa.

La relación entre variables de adversidad familiar y el desarrollo de depresión y otras dificultades en las adolescentes ha sido mostrada en diversas investigaciones, y parece estar influida por la percepción que tienen los adolescentes del soporte que su familia les brinda (Fendrich, Warmer \& Weissman, 1990). La percepción de soporte familiar ha sido vinculada a la frecuencia con que los padres escuchan el punto de vista de los hijos, la magnitud en que conocen lo que les está sucediendo a éstos, y la percepción que tienen los adolescentes acerca de que los padres son la principal fuente de ayuda cuando se está en problemas o frente a sentimientos de soledad (Helsen, Vollebergh \& Meeus, 2000). Un factor que hace compleja la evaluación de la influencia del soporte social familiar en la depresión es que la percepción de éste por parte del adolescente está también influida por su propio estado de ánimo, por lo que es de particular relevancia estimar la relación de esta variable en el tiempo con el desarrollo de sintomatología depresiva.

En síntesis, esta investigación considera tres variables que se han mostrado claramente relacionadas con la depresión en adolescentes, en especial en investigaciones transversales: estilo inferencial depresógeno, rumiación negativa y soporte familiar. Se evalúa en forma prospectiva la relación de las variables descritas con el desarrollo de síntomas depresivos en mujeres que se encuentran en la fase temprana de la adolescencia, etapa en que comienza un incremento de la sintomatología depresiva especialmente en el género femenino.

\section{Método}

\section{Participantes}

El estudio se realizó con una muestra de estudiantes de sexo femenino de enseñanza secundaria, que atienden población de nivel socioeconómico bajo y medio de la intercomuna Concepción-Talcahuano. Todas las participantes consintieron informadamente su participación en el estudio. Del total original de participantes 4 no lograron ser ubicadas en ninguna de las evaluaciones de seguimiento, por lo que la muestra definitiva empleada en los análisis fue de 142 participantes. No se observaron diferencias en los niveles de depresión inicial entre las participantes que se mantuvieron en el estudio respecto de las que no pudieron ser re-evaluadas. $\mathrm{Al}$ momento de la primera evaluación todas eran estudiantes de primer año de enseñanza secundaria y la gran mayoría (81\%) tenía 14 años (rango 13 a 17 años). Las participantes eran parte del grupo control de un estudio más amplio, razón por la cual la mitad habían sido tamizadas previamente y presentaban, 
como criterio de inclusión, puntajes en el BDI-II superiores a 7. Como criterio de exclusión se consideró la presencia de trastorno depresivo el último mes, lo que se midió con la entrevista estructurada DISC-IV (Bravo et al., 2001; Shaffer, Fisher, Lucas, Dulcan \& Schwab-Stone, 2000).

\section{Variables e instrumentos}

\section{Sintomatología depresiva}

Fue evaluada a través del Inventario de Depresión de Beck-Segunda Versión (BDI-II) (Beck, Steer \& Brown, 1996), instrumento que permite medir síntomas depresivos en adolescentes y adultos, con una edad mínima de 13 años, y que es ampliamente utilizado por sus cualidades psicométricas (Beck et al., 1996; Melipillán, Cova, Rincón \& Valdivia, 2008; Sanz, Navarro \& Vázquez, 2003). En la presente investigación su valor Alfa fue de 0.91.

\section{Soporte familiar}

Se empleó una escala tipo Likert de 7 ítems derivados de la Scale of Perceived Social Support (SPSS) desarrollada por Macdonald (1998). Los ítems seleccionados permiten cubrir los aspectos fundamentales del soporte familiar, siguiendo la propuesta de este autor: apoyo emocional, valoración del apoyo, apoyo informacional y apoyo instrumental. El Alfa observado en la presente investigación fue de 0.78 , semejante al obtenido por el autor para la escala original total. La correlación test retest a seis meses fue de 0.68.

\section{Estilo inferencial negativo}

Se evaluó con una versión abreviada del Adolescent Cognitive Style Questionaire, ACSQ (Hankin \& Abramson, 2002), instrumento que fue diseñado específicamente para medir las tres dimensiones del estilo inferencial negativo: atribución de causalidad (estabilidad y globalidad), inferencias negativas sobre las consecuencias, e implicancias negativas hacia sí mismo, proponiendo 12 situaciones hipotéticas relevantes para adolescentes respecto de las cuales el adolescente debe escoger el tipo de inferencias que cree realizaría. La consistencia interna de este instrumento encontrada por los autores fue de 0.95 , y la confiabilidad test-retest en un período de dos semanas fue de 0.73. Para la presente investigación se tradujo y se abrevió el instrumento (sólo 6 situaciones). El estudio piloto mostró que esta versión abreviada conservaba la consistencia interna del instrumento original. El Alfa de Cronbach observado en la presente investigación fue de 0.92 , y la correlación test retest a seis meses fue de 0.48 .

\section{Rumiación negativa}

La rumiación ha sido habitualmente evaluada con la Ruminative Response Scale, RRS (Treynor et al., 2003). Estudios recientes con esta escala han identificado dos subfactores de rumiación, uno de los cuales sería el que tiene una relación más relevante con el desarrollo de síntomas depresivos (Cova et al., 2007; Treynor et al., 2003). Este subfactor, al que se la ha traducido como rumiación negativa (brooding en inglés), es el que se consideró en el presente estudio. La escala RRS sólo tiene 5 ítems que miden este factor, por lo que, siguiendo las sugerencias de Treynor et al. (2003), se le amplió en dos ítems adicionales. El valor Alfa observado de la escala fue de 0.81 , y la correlación test retest a seis meses fue de 0.57 .

\section{Análisis estadísticos}

Se utilizó el programa Stata 12.0. Para analizar los cambios a lo largo del tiempo en los puntajes de depresión y los posibles efectos moderadores de dichos cambios por parte de predictores de nivel individual se empleó un modelo multinivel para el cambio (Singer \& Willet, 2003), que permite examinar el comportamiento de una variable a lo largo del tiempo y la relación de dicho comportamiento con otras variables (los predictores planteados). Una ventaja de este modelo para el análisis de datos longitudinales es que no requiere que cada participante tenga el mismo número de mediciones en la variable dependiente medida: puntajes de sintomatología depresiva obtenidos en la segunda, tercera y 
cuarta mediciones $(6,13$ y 24 meses desde la medición inicial). La medición inicial de sintomatología depresiva fue utilizada como covariable, a fin de controlar el efecto que los predictores analizados en este estudio pudieran mostrar con la depresión inicial. Como predictores de nivel 1 (intra-sujeto) se incluyó sólo el tiempo, mientras que como predictores de nivel 2 (entre-sujetos) se incluyeron soporte familiar, estilo inferencial y rumiación negativa.

La evaluación de los efectos fijos asociados a los predictores se llevó a cabo mediante un estadístico Z. Por su parte, la evaluación de los efectos aleatorios se realizó mediante un test de razón de verosimilitud (likelihood-ratio test), en el cual se compararon la calidad del ajuste a los datos de dos modelos que compartían los mismos efectos fijos, pero que diferían en que sólo uno de ellos incluía el efecto aleatorio evaluado.

Debido a que la variable dependiente del análisis (sintomatología depresiva) evidenciaba una marcada asimetría positiva, sus datos fueron normalizados empleando la transformación raíz cuadrada, modificación que permitió lograr un mejor cumplimiento de los supuestos de linealidad y normalidad inherentes al modelo.

\section{Resultados}

En la Tabla 1 se presentan los promedios y desviaciones estándar de las variables en las distintas mediciones (se presentan los valores originales, sin normalización).

El análisis se llevó a cabo especificando una secuencia de modelos de creciente complejidad.

TABLA 1

Valores descriptivos de las variables

\begin{tabular}{lccc}
\hline & $\mathrm{N}$ & $\mathrm{M}$ & $\mathrm{DE}$ \\
\hline Depresión $\mathrm{T}_{1}$ & 142 & 13.64 & 8.18 \\
Depresión $_{2}$ & 133 & 12.70 & 8.68 \\
Depresión $_{2}$ & 113 & 12.05 & 8.42 \\
Depresión $_{4}$ & 129 & 9.64 & 8.70 \\
Soporte familiar $\mathrm{T}_{1}$ & 142 & 10.53 & 2.74 \\
Estilo inferencial $\mathrm{T}_{1}$ & 142 & 2.10 & 0.97 \\
Rumiación negativa $\mathrm{T}_{1}$ & 142 & 9.42 & 4.72 \\
\hline
\end{tabular}

Fuente: elaboración propia.
En el paso 1 se procedió a estimar un modelo de intercepto aleatorio, donde el resultado obtenido para la estimación de la varianza intra-sujetos fue de $s^{2}=0.8$, mientras que para la estimación de la varianza entre-sujetos correspondió a $t=1.35$. En cuanto al coeficiente de correlación intraclase, éste indicó que el $62.7 \%$ de la varianza total en depresión correspondía a variabilidad entre-sujetos.

En el paso 2 se modeló la relación entre el tiempo (efecto lineal) y los puntajes de depresión, indicando la existencia de una tendencia a la disminución en los puntajes de depresión a lo largo del tiempo $(b=-0.029, p<0.001)$.

En el paso 3 se modificó el modelo anterior permitiendo que las trayectorias de depresión a lo largo del tiempo variaran aleatoriamente entre las participantes. Al comparar mediante un test de razón de verosimilitud este nuevo modelo, que incluía un efecto aleatorio para la pendiente del tiempo, con el modelo final del paso 2, que sólo incluía un efecto fijo para el tiempo, se obtuvo como resultado $\Delta D=6.34, p=0.012$, mostrando un mejor ajuste a los datos por parte del modelo que incluía el efecto aleatorio.

En el paso 4 se procedió a estimar la necesidad de incluir la covarianza entre los interceptos aleatorios y las pendientes aleatorias del modelo final del paso 3, para lo cual se llevó a cabo un test de razón de verosimilitud entre el modelo final del paso 3 y el modelo que incluía la covarianza entre los efectos aleatorios. El resultado correspondió a $\Delta D=1.09$, $p=0.30$, favoreciendo el modelo que no incluía el efecto de la covarianza.

Finalmente, en el paso 5, se procedió a incluir predictores de nivel individual (nivel 2), para dar cuenta de la varianza en el intercepto y en la pendiente de las trayectorias de depresión. Las variables incluidas en este paso correspondieron a soporte familiar, estilo inferencial y rumiación negativa, así como la interacción de cada una de ellas con el tiempo (véase Tabla 2, Modelo inicial). Siguiendo la recomendación de Singer y Willet (2003), los predictores de nivel 2 fueron centrados en la gran media (media de la muestra); junto a los anteriores predictores se incluyó la medición inicial de depresión como covariable. 
Al analizar el efecto de estos predictores sobre el promedio de depresión de los participantes a lo largo del tiempo (intercepto) se observó un efecto significativo por parte de la medición inicial de depresión $(b=0.371, p<0.001)$, del soporte familiar $(b=-0.096, p=0.013)$ y del estilo inferencial $(b=0.235, p=0.045)$. La rumiación negativa no mostró una relación estadísticamente significativa. Estos resultados permiten concluir que los mayores niveles de soporte familiar se asocian a menores niveles promedio de depresión a lo largo del seguimiento, mientras que el estilo inferencial depresógeno se asocia a mayores niveles promedio de depresión a lo largo del tiempo.

En cuanto a los efectos de los tres predictores sobre la pendiente asociada al tiempo (trayectoria), los resultados evidenciaron que ninguno de ellos mostró una relación estadísticamente significativa, indicando que la tasa de cambio de la depresión asociada al tiempo no se ve afectada por ninguno de los predictores analizados.

Dados los resultados anteriores, se estimó un modelo final que sólo incluía como predictores de nivel 2 el efecto de la depresión inicial, soporte fa- miliar y estilo inferencial sobre los valores iniciales de depresión (Tabla 2, Modelo final). El resultado obtenido mostró una relación estadísticamente significativa de los tres predictores con el intercepto, indicando que el promedio de depresión a lo largo del tiempo se relaciona con los predictores, así: depresión inicial evidenció una relación directa $(b=0.406, p<0.001)$; soporte familiar evidenció una relación inversa $(b=-0.095, p=0.004)$, y estilo inferencial evidenció una relación directa $(b=0.324, p<0.001)$.

El anterior análisis fue realizado tratando el estilo inferencial negativo como si fuera equivalente al promedio de las tres dimensiones que conforman esta variable. Sin embargo, algunos autores han propuesto que lo más apropiado no es considerar este valor promedio, sino el valor más alto que cada sujeto obtiene en cualquiera de las dimensiones, dado que se trataría del aspecto de mayor vulnerabilidad, el "eslabón más débil" (Abela \& Sarín, 2002). Repetido el análisis anterior, tratando de este modo la variable estilo inferencial negativo, los resultados obtenidos fueron los mismos.

TABLA 2

Resultados modelos multinivel para el cambio en los niveles de sintomatología depresiva

\begin{tabular}{|c|c|c|c|c|}
\hline & \multirow{2}{*}{ Coef. } & \multirow{2}{*}{$\mathrm{EE}$} & \multicolumn{2}{|c|}{ IC 95\% } \\
\hline & & & L. Inf. & L. Sup. \\
\hline \multicolumn{5}{|l|}{ Nivel 1: (Modelo final) } \\
\hline Intercepto & $3.420 * * *$ & 0.093 & 3.238 & 3.602 \\
\hline Tiempo (lineal) & $-0.03 * * *$ & 0.008 & -0.045 & -0.015 \\
\hline \multicolumn{5}{|l|}{ Nivel 2: Modelo inicial } \\
\hline Depresión pretest & $0.371 * * *$ & 0.092 & 0.190 & 0.552 \\
\hline Soporte familiar & $-0.096^{*}$ & 0.039 & -0.171 & -0.02 \\
\hline Estilo inferencial & $0.235 *$ & 0.117 & 0.006 & 0.463 \\
\hline Rum. Negativa & 0.235 & 0.185 & -0.128 & 0.597 \\
\hline Soporte familiar $\times$ Tiempo & 0.002 & 0.003 & -0.004 & 0.008 \\
\hline Estilo inferencial $\times$ Tiempo & 0.008 & 0.009 & -0.010 & 0.027 \\
\hline Rum. Negativa $\times$ Tiempo & -0.010 & 0.014 & -0.039 & 0.018 \\
\hline \multicolumn{5}{|l|}{ Nivel 2: Modelo Final } \\
\hline Depresión pretest & $0.406 * * *$ & 0.088 & 0.233 & 0.578 \\
\hline Soporte familiar & $-0.095 * *$ & 0.033 & -0.16 & -0.03 \\
\hline Estilo inferencial & $0.324 * * *$ & 0.097 & 0.133 & 0.514 \\
\hline
\end{tabular}

$* p<0.05 ; * p<0.01 ; * * *<0.001$

Fuente: elaboración propia. 


\section{Discusión}

Las variables que se consideraron en el presente estudio han mostrado en diversas investigaciones estar correlacionadas con la presencia de sintomatología depresiva. Sin embargo, dada la naturaleza transversal de muchos de estos estudios, no es claro si esta relación se debe a que constituyen factores que influyen en el desarrollo de la depresión o son sólo correlatos de ésta.

La presente investigación mostró relación del soporte familiar y el estilo inferencial depresógeno con el promedio de sintomatología depresiva de los participantes a lo largo del periodo de 24 meses de seguimiento. Dado el control ejercido sobre la sintomatología depresiva inicial de los participantes y las mediciones prospectivas realizadas, esta relación indica que dichas variables tienen un rol en la sintomatología depresiva no atribuible a ser solamente correlatos o consecuencias de la depresión. No se observó, sin embargo, que estas variables incrementen la tendencia a desarrollar sintomatología depresiva, indicando que probablemente otros factores deben interactuar para generar este efecto.

La percepción de menor soporte provisto por la familia se confirma como factor que contribuye a la presencia de depresión en las adolescentes. Estudios recientes han mostrado relación del soporte familiar tanto con la sintomatología depresiva como con la presencia de conductas de auto-daño y la ideación suicida, particularmente en adolescentes de sexo femenino (Kerr, Preuss \& King, 2006; Tuisku et al., 2009). Por su parte, la relación observada entre estilo inferencial depresógeno con sintomatología depresiva está en concordancia con lo propuesto desde la teoría de la depresión por desesperanza, donde es considerado el factor de vulnerabilidad más relevante.

La importancia del estilo inferencial en el desarrollo de depresión ha tenido significativo respaldo en la investigación en la última década (Abela \& Hankin, 2008; Abela \& Sarin, 2002), aun cuando la falta de instrumentos con adecuadas propiedades psicométricas para evaluar este factor en la niñez y adolescencia ha dificultado la investigación en estas etapas de la vida. La versión abreviada del instrumento que se tradujo y adaptó para este estudio corroboró sus adecuadas propiedades psicométricas y el interés de este constructo.

La focalización repetitiva en la experiencia del sentimiento negativo y las lamentaciones acerca de su ocurrencia, con un componente autoinculpatorio, características de la denominada rumiación negativa (brooding), no mostró la esperada relación con la presencia y desarrollo de sintomatología depresiva (Nolen-Hoeksema, Stice, Wade, \& Bohon, 2007), lo que no es atribuible solamente al control ejercido de la sintomatología depresiva inicial dado que, pese a que esta característica hace más exigente la observación de un efecto, existen estudios análogos que sí la han observado (Abela \& Hankin, 2011; Cova et al., 2009). En el único estudio que se logró identificar dónde se evaluó conjuntamente rumiación negativa y estilo inferencial negativo como predictores (junto con otras variables e interacciones) se observó que la rumiación tenía relación con sintomatología depresiva a nivel del intercepto y no de la trayectoria de sintomatología depresiva, en cambio el estilo inferencial negativo mostró relación con la trayectoria y no con el intercepto (Hankin, 2009). En ese estudio no se controló la sintomatología depresiva inicial ni se incluyó soporte familiar, por lo que no es enteramente comparable al presente.

La ausencia de efecto de la rumiación negativa plantea interrogantes, lo que podría ser un efecto de la varianza compartida entre las variables consideradas. Otro factor influyente puede ser que la muestra estudiada presentaba un promedio de sintomatología inicial más elevado por lo que al ser controlado ese factor pudo haberse anulado el efecto de la rumiación. Es necesaria una mayor investigación al respecto dada la relevancia que se le asigna actualmente a la rumiación como factor depresógeno (Vázquez et al., 2010).

La tendencia observada a la disminución de los síntomas depresivos en las sucesivas mediciones no es coherente con las evidencias existentes respecto a que los síntomas y trastornos depresivos se incrementan entre los 13 y 18 años; sin embargo este fenómeno ha sido observado en muchos estu- 
dios de seguimiento, tanto de población general adolescente como de población adolescente con sintomatología subumbral (Abela \& Skitch, 2007; Auerbach, Eberhart \& Abela, 2009; Hankin, 2008, 2009; Yang et al., 2010). La disminución de la sintomatología depresiva en los grupos con niveles de sintomatología inicial elevados, como la existente en el presente estudio, podría explicarse por el fenómeno de regresión a la media. A nivel individual, la depresión adolescente es un fenómeno de mediana duración (Dozois \& Westra, 2004; Lewinsohn et al., 1998), por lo que es posible que muchas de las adolescentes que presentan niveles aumentados de sintomatología depresiva mejoren su nivel de adaptación al seguimiento. Otra alternativa de explicación es el acostumbramiento a los instrumentos, sin embargo ello es más probable que ocurra en periodos cortos de reevaluación que en el caso del presente estudio. Curiosamente, este fenómeno ha sido muy poco analizado.

El diseño prospectivo de la presente investigación permitió el empleo de los poderosos procedimientos de análisis longitudinales multinivel, que recién están utilizándose en la investigación en esta área. Junto con esta fortaleza, el estudio tiene dos de limitaciones importantes: una, que solo se emplearon instrumentos de autoinforme, y, dos, que no se evaluó la interacción de las variables consideradas con acontecimientos vitales negativos vividos por las adolescentes. La mayoría de los modelos explicativos de la depresión que consideran las variables estudiadas proponen que estos factores interactúan con circunstancias vitales negativas y que no necesariamente influyen en ausencia de ellas (Sanz \& Vázquez, 2008). El estilo de afrontamiento y la rumiación en interacción con eventos vitales ha mostrado relación tanto con el promedio de sintomatología a lo largo de tiempo como en la trayectoria en los pocos estudios realizados que han utilizado modelos longitudinales multinivel (Hankin, 2009). Pese a estas limitaciones, el estudio es de interés para la comprensión de los factores que inciden en el desarrollo de sintomatología depresiva en las adolescentes. La investigación al respecto en Latinoamérica es poca, y los resultados obtenidos sugieren la relevancia de que el soporte familiar y variables centrales de procesamiento cognitivo, como el estilo inferencial, sean consideradas en los programas de prevención y tratamiento de la depresión.

\section{Referencias}

Abela, J. R. Z. \& Hankin, B. L. (2008). Cognitive vulnerability to depression in children and adolescents. En J. R. Z. Abela \& B. L. Hankin (Eds.), Handbook of Depression in Children and Adolescents (pp. 35-78). New York, NY: The Guilford Press.

Abela, J. R. Z. \& Hankin, B. L. (2009). Cognitive vulnerability to depression in adolescents: A developmental psychopathology perspective. En S. Nolen-Hoeksema \& Lori M. Hilt (Eds.), Handbook of Depression in Adolescent (pp. 335-376). New York: Routledge Guilford Press.

Abela, J. R. Z. \& Hankin, B. L. (2011). Rumination as a vulnerability factor to depression during the transition from early to middle adolescence: A multiwave longitudinal study. Journal of Abnormal Psychology, 120, 259-271.

Abela, J. R. Z. \& Sarin, S. (2002). Cognitive vulnerability to hopelessness depression: A chain is only as strong as its weakest link. Cognitive Therapy and Research, 26, 811-829.

Abela, J. R. Z. \& Seligman, M. (2000). The hopelessness theory of depression: A test of the Diathesis-Stress component in the interpersonal and achievement domains. Cognitive Therapy and Research, 24, 361-378.

Abela, J. R. Z. \& Skitch, S. A. (2007). Dysfunctional attitudes, self-esteem, and hassles: Cognitive vulnerability to depression in children of affectively ill parent. Behaviour Research and Therapy, 45, 1127-1140.

Abramson, L. Y., Seligman, M. E. P. \& Teasdale, J. (1978). Learned helplessness in humans: Critique and reformulation. Journal of Abnormal Psychology, 87, 49-74.

Abramson, L., Metalsky, G. I. \& Alloy, L. B. (1989). Hopelessness depression: A theory-based subtype of depression. Psychological Review, 96, 358-372. 
Angold, A. \& Costello, E. (2001). The epidemiology of depression in children and adolescent. En I. M. Goodyer (Ed.), The Depressed Child and Adolescent (pp. 143-148). Cambridge: Cambridge University Press.

Auerbach, R. P., Eberhart, N. K. \& Abela, J. R. Z. (2008). Cognitive Vulnerability to Depression in Canadian and Chinese Adolescents. Journal of Abnormal Child Psychology, 38, 57-68.

Beck, A., Steer, R. \& Brown, G. (1996). Beck Depression Inventory. San Antonio: Psychological Corporation.

Bravo, M., Ribera, J., Rubio-Stipec, M., Canino, G., Shorout, P., Ramírez, R., et al., (2001). Test-retest reliability of the Spanish version of the Diagnostic Interview Schedule for Children (DISC-IV). Journal of Abnormal Child Psychology, 29, 433-444.

Brent, D. \& Weersing, V. R. (2008). Depressive disorders in Childhood and Adolescente. En M. Rutter, D. Bishop, D. Pine, S. Scott, J. Stevenson, E. Taylor \& A. Thapar (Eds.), Rutter's Child and Adolescent Psychiatry 5르. Ed. (pp. 587-612). Oxford: Blackwell Publishing Science.

Cova, F., Rincón, P. \& Melipillán, R. (2007). Rumiación y presencia de sintomatología ansiosa y depresiva en adolescentes. Revista Mexicana de Psicología, 24, 175-183.

Cova, F., Rincón, P. \& Melipillán, R. (2009). Reflexión, rumiación negativa y desarrollo de sintomatología depresiva en adolescentes de sexo femenino. Terapia Psicológica, 27, 155-160.

Dozois, D. J. A. \& Westra, H. A. (2004). The nature of anxiety and depression: implications for prevention. En D. J. A. Dozois \& K. S. Dobson (Eds.), The Prevention of Anxiety and Depression: Theory, Research and Practice (pp. 9-41). Washington, DC: American Psychological Association.

Fendrich, M., Warmer, V. \& Weissman, M. M. (1990). Family risk factors, parental depression, and psychopathology in offspring. Developmental Psychology, 26, 40-50.

Hankin, B. L. (2008). Rumination and depression in adolescence: Investigating symptom specificity in a multiwave prospective study. Journal of Clinical Child and Adolescent Psychology, 37, 701-713.
Hankin, B. L. (2009). Development of sex differences in depressive and co-ocurring anxious symptoms during adolescence: Descriptive trajectories and potential explanations in a multiwave prospective study. Journal of Clinical Child and Adolescent Psychology, 38, 460-472.

Hankin, B. \& Abramson, L. (2002). Measuring cognitive vulnerability to depression in adolescence: Reliability, validity, and gender differences. Journal of Clinical Child and Adolescent Psychology, 31, 491-504.

Hankin, B. L., Abramson, L., Moffitt, T., Silva, P., Mcgee, R. \& Angell, K. (1998). Development of depression from preadolescence to young adulthood: Emerging gender differences in a 10 year longitudinal study. Journal of Abnormal Psychology, 107, 128-140.

Hankin, B. L., Wetter, E. \& Cheely, C. (2008). Sex differences in Child and Adolescent Depression: A Developmental Psychoptahological Approach. En J. R. Z. Abela \& B. L. Hankin (Eds.), Handbook of Depression in Children and Adolescents (pp. 377414). New York, NY: The Guilford Press.

Helsen M., Volleberg, W. \& Meeus, W. (2000). Social support from parents and friends and emotional problems. Journal of Youth and Adolescence, 29, 319-335.

Hilt, L. \& Nolen-Hoeksema, S. (2009). The emergence of gender differences in depression in adolescence. En S. Nolen-Hoeksema \& L. M. Hilt (Eds.), Handbook of Depression in Adolescent (pp. 111-136). New York: Routledge Guilford Press.

Hyde, J., Mezulis, A. \& Abramson, L. (2008). The ABCs of depression: Integrating affective, biological, and cognitive models to explain the emergence of gender difference in depression. Psychological Review, 115, 291-313.

Kerr, D. C. R., Preuss, L. J. \& King, C. A. (2006). Suicidal adolescents' social support from family and peers: Gender-specific associations with psychopathology. Journal of Abnormal Child Psychology, 34, 103-114.

Lewinsohn, P. M., Rohde, P. \& Seeley, J. R. (1998). Major depressive disorder in older adolescents: Prevalence, risk factors, and clinical implications. Clinical Psychology Review, 18, 765-794. 
Macdonald, G. (1998). Development of a Social Support Scale: A evaluation of psychometric properties. Research on Social Work Practice, 8, 546-576.

Melipillán, R., Cova, F., Rincón, P. \& Valdivia, M. (2008). Propiedades psicométricas del Inventario de Depresión de Beck II en adolescentes chilenos. Terapia Psicológica, 26, 59-69.

Murray, C. J. \& López, A. D. (1998). The global burden of disease, 1990-2020. Nature Medicine, 4, 1241-1243.

Nolen-Hoeksema, S. (2000). The role of rumination in depressive disorders and mixed anxiety/depressive symptoms. Journal of Abnormal Psychology, 109, 504-511.

Nolen-Hoeksema, S., Stice, E., Wade, E. \& Bohon, C. (2007). Reciprocal relations between rumination and bulimic, substance abuse, and depressive symptoms in female adolescents. Journal of Abnormal Psychology, 116, 198-207.

Sanz, J. \& Vázquez, C. (2008). Trastornos del estado de ánimo: Teorías psicológicas. En A. Belloch, B. Sandín \&. F. Ramos (Eds.), Manual de Psicopatología (pp. 271-298). Madrid: McGraw Hill. Edición revisada.

Sanz, J., Navarro, M. E. \& Vázquez, C. (2003). Adaptación española del inventario para la depresión de Beck-II (BDI-II): 1. Propiedades psicométricas en estudiantes universitarios. Análisis y Modificación de Conducta, 29, 239-288.

Shaffer, D., Fisher, P., Lucas, C. P., Dulcan, M. K. \& Schwab-Stone, M. E. (2000). NIMH Diagnostic Interview Schedule for Children Version IV (NIMH DISC-IV): description, differences for previous version and reliability of some common diagnoses. Journal of American Academy of Child and Adolescent Psychiatry, 39, 28-38.
Singer, J. D. \& Willet, J. B. (2003). Applied Longitudinal Data Analysis. New York: Oxford University Press.

Southall, D. \& Roberts, J. E. (2002). Attributional style and negative affect among adolescent depressive symptoms following life stress: A 14 week prospective study. Cognitive Therapy and Research, 26, 563-579.

Treynor, W., González, R. \& Nolen-Hoeksema, S. (2003). Rumination reconsidered: A psychometric analysis. Cognitive Therapy and Research, 27, 247-259.

Tuisku, V., Pelkonen, M., Kiviruusu, O., Karlsson, L., Ruuttu, T. \& Marttunen, M. (2009). Factors associated with deliberate self-harm behaviour among depressed adolescent outpatients. Journal of Adolescence, 32, 1125-1136.

Üstun, T. B., Ayuso-Mateos, J. L., Chatterji, S. \& Mathers, C. (2004). Global burden of depressive disorders in the year 2000. British Journal of Psychiatry, 184, 386-392.

Vázquez, C., Hervás, G., Hernangómez, L. \& Romero, N. (2010). Modelos cognitivos de la depresión: Una síntesis y nueva propuesta basada en 30 años de investigación. Behavioral Psychology/Psicología Conductual, 18, 139-165.

Wade, T., Cairney, J. \& Pevalin, D. (2002). Emergence of gender differences in depression during adolescence: National panel results from three countries. Journal of American Academy of Child and Adolescent Psychiatry, 41, 190-198.

Yang, J., Yao, Y., Zhu, Z., Zhang, C., Ling, Y., Abela, J. R. Z., et al., (2010). The impact of stress on depressive symptoms is moderated by social support in Chinese adolescents with subthreshold depression: A multi-wave longitudinal study. Journal of Affective Disorders, 127, 113-121. 